\title{
AGN Evolution from Large and Deep X-Ray Surveys
}

\author{
Marcella Brusa ${ }^{1}$ \\ ${ }^{1}$ Max Planck Institute für Extraterrestrische Physik, \\ Giessenbachstrasse, 1 DE-85748, garching bei München, Germany \\ Email: marcella@mpe.mpg.de
}

\begin{abstract}
Over the last few years, the existence of mutual feedback effects between accreting supermassive black holes powering AGN and star formation in their host galaxies has become evident. This means that the formation and the evolution of AGN and galaxies should be considered as one and the same problem. As a consequence, the search for, and the characterization of the evolutionary and physical properties of AGN over a large redshift interval is a key topic of present research in the field of observational cosmology. Significant advances have been obtained in the last ten years thanks to the sizable number of $X M M-N e w t o n$ and Chandra surveys, complemented by multiwavelength follow-up programs. I will present some of the recent results and the ongoing efforts (mostly from the COSMOS and CDFS surveys) aimed at obtaining a complete census of accreting black holes in the universe, and a characterization of the properties of the host galaxies.
\end{abstract}

Keywords. galaxies: active, galaxies: starburst, X-rays: galaxies

Since the 1999 launches of the XMM-Newton and Chandra satellites, a large $(>30)$ number of surveys covering a wide fraction of the area vs. depth plane (see Figure 1 of Brandt \& Hasinger 2005; see also Hickox 2009) have been performed and our understanding of AGN properties and evolution has received a major boost. Thanks to vigorous programs of multiwavelength follow-up campaigns which, in the last few years, have became customary, sensitive X-ray observations have been highly efficient in unveiling weak and/or elusive accreting black holes, in a variety of otherwise non-active galaxies, such as (among others): X-ray bright optically normal galaxies (XBONGs, Comastri et al. 2002), extremely red objects (e.g., Brusa et al. 2005), submillimeter galaxies (e.g., Alexander et al. 2005) high- $z$ star-forming systems (e.g., Daddi et al. 2007; Fiore et al. 2008). In many of these cases, the AGN responsible for the X-ray emission is overwhelmed at longer wavelengths by the host-galaxy light and/or the obscuration might be connected to processes within the host galaxy itself, such as the star-formation rate and the presence of dust lanes or starburst disks (see, e.g., Hopkins et al. 2009). This suggests that the accretion activity (especially in high-redshift sources) is unambiguously revealed thanks to the presence of a strong X-ray emission (see e.g., discussion by Brusa et al. 2009a) and therefore, the combination of both X-ray and optical classifications can be crucial to fully assess the nature of the candidate AGN.

The high level of completeness in redshift determination for a large number of X-ray selected AGN (up to a few thousand) has made possible a robust determination of the luminosity function and evolution of unobscured and mildly obscured AGN that turned out to be luminosity dependent: the space density of bright QSOs $\left(L_{\mathrm{X}}>10^{44} \mathrm{erg} \mathrm{s}^{-1}\right)$ peaks at $z \sim 2-3$, to be compared with the $z \sim 0.7-1$ peak of lower luminosity Seyfert galaxies (Ueda et al. 2003; La Franca et al. 2005; Silverman et al. 2008a; Ebrero et al. 2009). Based on these works, Marconi et al. (2004) and Merloni (2004) were the first to 
propose that SMBH undergo an "anti-hierarchical" evolution, in the form of differential growth (earlier and faster for larger black holes). This anti-hierarchical behavior observed in AGN evolution (similar to that observed in normal galaxies, e.g., Cowie et al. 1996) provided an important, independent, confirmation that the formation and evolution of SMBH and their host galaxies are likely different aspects of the same astrophysical problem. In particular, accretion onto supermassive black holes triggered by galaxies mergers and/or collisions may provide the "feedback" needed in almost all recent models for galaxy formation and evolution in order to recover the properties (masses, luminoisity, clustering) we observe today in local systems.

In this general framework, the differences between "obscured" and "unobscured" AGN are no longer uniquely described under a zeroth-order geometrical unification model (in which they are simply related to orientation effects; Antonucci \& Miller 1985), but can be interpreted as due to the fact that the same object is observed in different evolutionary phases. This hypothesis is further supported by the finding that absorption is much more common at low luminosities (Ueda et al. 2003; Maiolino et al. 2007) and at high redshift (La Franca et al. 2005; Treister \& Urry 2006; Hasinger 2008), as demonstrated by results from X-ray surveys. The luminosity and redshift dependence of the obscuring fraction may be naturally linked to the AGN radiative power (related to the intrinsic $\mathrm{X}$-ray luminosity) which is able to ionize and expel gas and dust (more common at high-

$z$ ) from the nuclear regions, nicely fitting the current framework of AGN formation and evolution sketched above (see, for example, Hopkins et al. 2006 and these proceedings).

The complete picture is likely to be much more complicated, depending on many other parameters (such as, e.g., the BH mass, the Eddington ratio, the QSO duty cycle), and in particular related to the complex "light curves" of AGN, which, in turn, depend on the detailed hydrodynamics adopted in the simulations. A correct and complete identification of unobscured, obscured and highly obscured AGN at all redshifts (especially in the $z=1-3$ interval, where most of the feedback is expected to happen) is therefore crucial for a comprehensive understanding of the still little-explored phase of the common growth of SMBHs and their host galaxies.

Here I highlight recent results of the characterization of the high-luminosity tail of the X-ray luminosity function from the COSMOS survey, and on the host-galaxy properties of obscured AGN at cosmological distances, from an analysis of X-ray selected sources in the Chandra Deep Field South (CDFS). I also present evidence for evolution of the $M_{\mathrm{BH}}-M_{\text {bulge }}$ relation as derived from optically selected BL AGN from the zCOSMOS survey (almost all of them are also X-ray emitters).

\section{The High-Luminosity Tail of X-Ray Selected AGN: Results from the COSMOS Survey}

The high completeness in optical identifications of the $X M M-$ COSMOS sample (Brusa et al. 2009b) and the availability of spectroscopic and photometric redshifts (Salvato et al. 2009) allows us to estimate the number densities of AGN as a function of luminosity and redshift. In calculating the binned number-density evolution, we considered only the subsample of sources detected in the hard $(2-10 \mathrm{keV})$ band and included in the flux-limited sample (925 sources at $S_{\mathrm{x}}=6 \times 10^{-15} \mathrm{erg} \mathrm{s}^{-1} \mathrm{~cm}^{-2}$, and $\sim 60 \%$ with spectroscopic redshifts available). Since the hardness ratios between the hard and soft bands allows an estimate of the absorption column density, we present the XLF of the deabsorbed $2-10 \mathrm{keV}$ luminosity. In our first analysis, we use the traditional $1 / V_{\text {a }}$ estimator 
(Avni \& Bahcall 1980). Additional details on the method used in deriving the XLF are given by Brusa et al. (in preparation) and Miyaji et al. (in preparation).

We compared our first estimates of the number-density evolution of the most luminous AGN population with recent measurements. The results are presented in Figure 1, where the number density of $X M M-$ COSMOS sources is plotted against the redshift in the luminosity bin $\log L_{\mathrm{X}}=44.5-47$ (squares). We only included the bins where the lowest$L_{\mathrm{X}}$, lowest- $z$ boundary corresponds to a $2-10 \mathrm{keV}$ flux above our limit for plotting. In the same figure, we plot the data points obtained from the AEGIS survey by Aird et al. (2009; circles), along with the best-fit luminosity function (black curve). The results, based on six datapoints in the range $z=1-3$ with $\sim 20$ objects each, may provide robust constraints on the shape of the XLF. In particular, the $X M M-$ COSMOS data points seem to favor a higher redshift $(z \sim 2)$ peak for the space density of luminous quasars as predicted by a LDDE parameterization, represented in Figure 1 by the Ebrero et al. (2009, short-dashed curve) and the Silverman et al. (2008, long-dashed curve) luminosity functions, though different in the details, and reproduced by XRB synthesis models (Gilli, Comastri \& Hasinger 2007; dot-dashed curve) rather than the lower redshift $(z \sim 1)$ peak expected from the LADE model recently proposed by Aird et al. (2009).

\section{Black Hole and Star-Formation Activity at $z>1$}

In order to study the host galaxies of obscured AGN, we defined a sample of 116 "bona fide" obscured AGN, detected in the 1Ms CDFS observation (Alexander et al. 2003) and for which deep infrared (IRAC and MIPS) $K$-band and multiwavelength optical photometry is available (from Grazian et al. 2006, the MUSIC survey). We selected sources without broad lines in the optical spectra and with small optical nuclear emission with respect to the host galaxy optical emission.

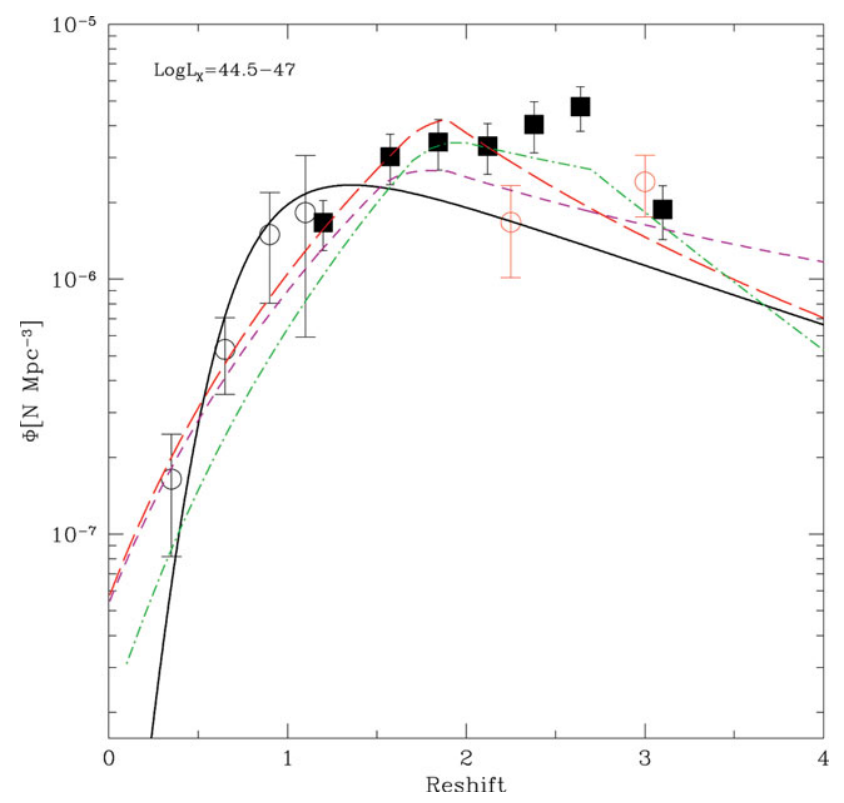

Figure 1. The number density evolution from the $X M M-$ COSMOS 53 field AGN (squares) compared with recent results from Aird et al. (2009; circles, and black solid line), Ebrero et al. (2009; short-dashed curve), Silverman et al. (2008; long-dashed curve), and the expectation of XRB synthesis models taken from Gilli et al. (2007, dot-dashed curve). 
We found that the hosts of obscured AGN are redder in the $U-V$ rest-frame than the overall galaxy population at the same redshift: in particular, obscured AGN mainly populate the red sequence and the green valley in the color-magnitude plots, in agreement with the results of Silverman et al. (2008b). For the MUSIC sample, the $U-V$ galaxy colors are strongly correlated with the $K$-band absolute magnitude (Figure 2 ), and therefore with the galaxy stellar mass, with the most massive systems having a redder color. The hosts of the obscured AGN are therefore found in the red-massive tail of the distribution of optically selected galaxies in all three redshift bins we considered (see labels in the figure). AGN feedback is often invoked as one of the main explanations of the observed galaxy colors (e.g., Nandra et al. 2007; Hasinger 2008). However, it is well known that the main ingredient for nuclear activity is the presence of SMBHs in galactic nuclei, and that SMBHs are found almost exclusively in massive galaxies (e.g., Magorrian et al. 1998). Therefore, it is not truly surprising to find AGN hosted in massive galaxies, and the simple presence of AGN in massive red galaxies is not enough to argue for a significant feedback effect on the observed colors because of the strong color-mass correlation. If AGN feedback was responsible for the observed red colors, since galaxy colors are strongly correlated with the galaxy mass and AGN are found preferably in massive galaxies, then AGN feedback would be considered one of the main players in the building of the galaxy mass-color correlation.

We found that about $2 / 3$ of the obscured AGN hosts at all redshifts show substantial (>10 $M_{\odot} \mathrm{yr}^{-1}$ ) star-formation activity (Figure 3, where two histograms of the SFR derived from SED fitting are shown, for optical and infrared selected samples) and about half live in galaxies which are still actively forming stars. For these sources, the observed

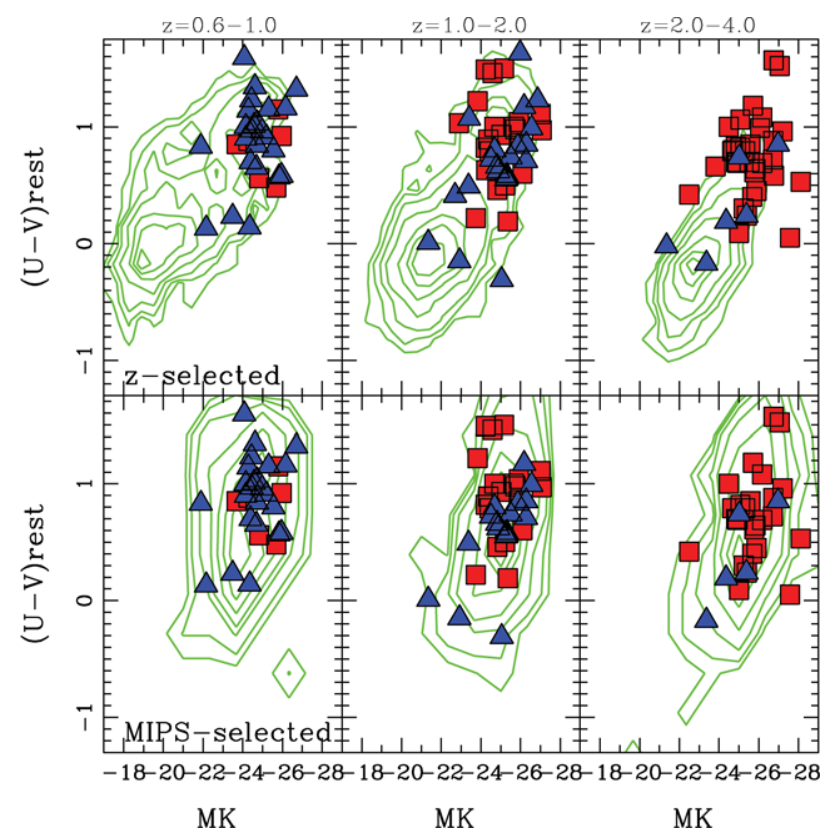

Figure 2. $U-V$ (rest frame) vs. the absolute $K$-band magnitude for obscured AGN (squares and triangles) and the underlying galaxy population (contours). The upper panels show a comparison of the X-ray selected sources with the optically selected ( $z$-band) galaxy population in three different redshift bins: $z=0.6-1.0, z=1-2$, and $z=2-4$ from left to right, respectively. The lower panels show the comparison for the subsample of objects detected also at $24 \mu \mathrm{m}$, in the same redshift bins. 
red colors are likely due to dust extinction rather than an evolved stellar population. We then conclude that a significant fraction of obscured AGN live in massive, dusty star-forming galaxies with red optical colors.

We compared the number of obscured AGN and of all X-ray selected AGN to the number of field galaxies in broad bins of galaxy stellar mass $\left(M_{*}=10^{10}-10^{12} M_{\odot}\right)$ and redshifts $(z=0.6-1, z=1-2, z=2-4)$. We find that the AGN fraction increases with the host galaxy stellar mass, from $\sim 1 \%$ at $M_{*} \sim 10^{10} M_{\odot}$ to $\sim 30 \%$ at $M_{*} \sim$ $3 \times 10^{11} M_{\odot}$ (see also Yamada et al. 2009), and the actual trend of increasing AGN fraction as a function of the stellar mass is probably steeper given the uncompleteness of the MUSIC sample at $M_{*}<10^{11} M_{\odot}$ (see Figure 4$)$. We compared this trend with that observed in the local universe (Best et al. 2005) for AGN with luminosities above similar thresholds. While the observed trend is the same in all the redshift bins investigated, the AGN fraction is higher than that observed in the local universe, and it could be much higher. In fact, we are comparing AGN selected with two different methods: forbidden emission-line luminosity (SDSS) and X-ray emission (GOODS). The latter sample does not contain most Compton-thick AGN. On the other hand, Compton-thick AGN may well be present in [O III]-selected AGN samples. The fraction of Compton-thick AGN not directly detected in deep Chandra surveys is estimated to be between $40 \%$ and $100 \%$ of the X-ray selected AGN, using infrared selection or other techniques (see Donley et al. 2008; Fiore et al. 2008, and references therein). Therefore, under the simplest assumption that this Compton-thick AGN fraction is constant with galaxy mass, the discrepancy observed in Figure 4 could increase by as much as a factor of two.

The fraction of active galaxies in the total galaxy population is proportional to the AGN duty cycle. Our results would thus suggest higher AGN duty cycles at $z=2-4$ than at $z=0$, in agreement with expectations from most recent semi-analytic models

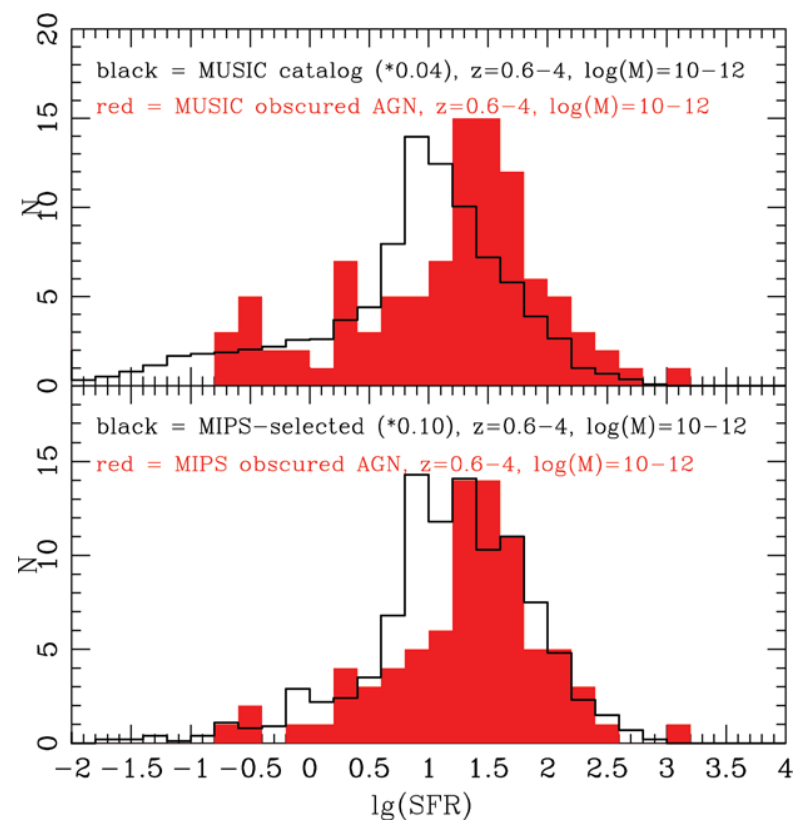

Figure 3. The distribution of the SFR for both the optically (upper) and MIPS (lower) selected sample of field galaxies (open histogram) and obscured AGN (filled histogram) in the redshift interval $z=1-4$ and in a specific stellar mass range $\left(M_{*}=10^{10}-10^{12} M_{\odot}\right)$. 
(e.g., Menci et al. 2008), in which AGN activity is present in a larger fraction of galaxies at higher redshift.

The fact that the most luminous obscured AGN are found in the most massive galaxies at all redshifts may suggest that $L / L_{\mathrm{Edd}}$ of the obscured AGN is similar, particularly in the case of the most luminous sources $\left(\log L_{\mathrm{X}}>43 \mathrm{erg} \mathrm{s}^{-1}\right)$, for which the luminosity threshold introduces a bias against the sources accreting at lower rates in the lowest redshift bin. Assuming the local Magorrian relation between $M_{\mathrm{BH}}$ and $M_{*}$ (e.g., Marconi \& Hunt 2003) and a bolometric correction of 20 (e.g., Marconi et al. 2004) the median observed values of $L_{\mathrm{X}} / M_{*}$ correspond to $L / L_{\mathrm{Edd}} \sim 0.1$. Although it suffers from large uncertainties associated with the stellar mass and $\mathrm{BH}$ mass estimates, this value can be considered as representative of the accretion state of the most luminous obscured AGN in the present sample. Similar results are obtained for Chandra Deep Field North X-ray sources at $z=2-4$ (Yamada et al. 2009) and are also typical of unobscured type 1 AGN at $z>1$ (see Merloni et al. 2009).

\section{The Evolution of the $M_{\mathrm{BH}}-M_{\text {bulge }}$ Relation}

Local scaling relations have proved themselves unable to unambiguously determine the physical nature of the SMBH-galaxy coupling. A large number of theoretical models for the AGN-galaxy interaction have been proposed, all tuned to reproduce the $z=0$ observations. One obvious way out of this impasse is the study of the evolution of scaling relations, which had until recently been limited to a handful of objects in narrow redshift windows (Peng et al. 2006 and these proceedings; Woo et al. 2008 and these proceedings; Jahnke et al. 2009 and these proceedings).

Within the zCOSMOS collaboration, a massive spectroscopic campaign with $\sim 10,000$ spectra of galaxies and AGN (Lilly et al. 2007), we have pioneered a new method to unveil

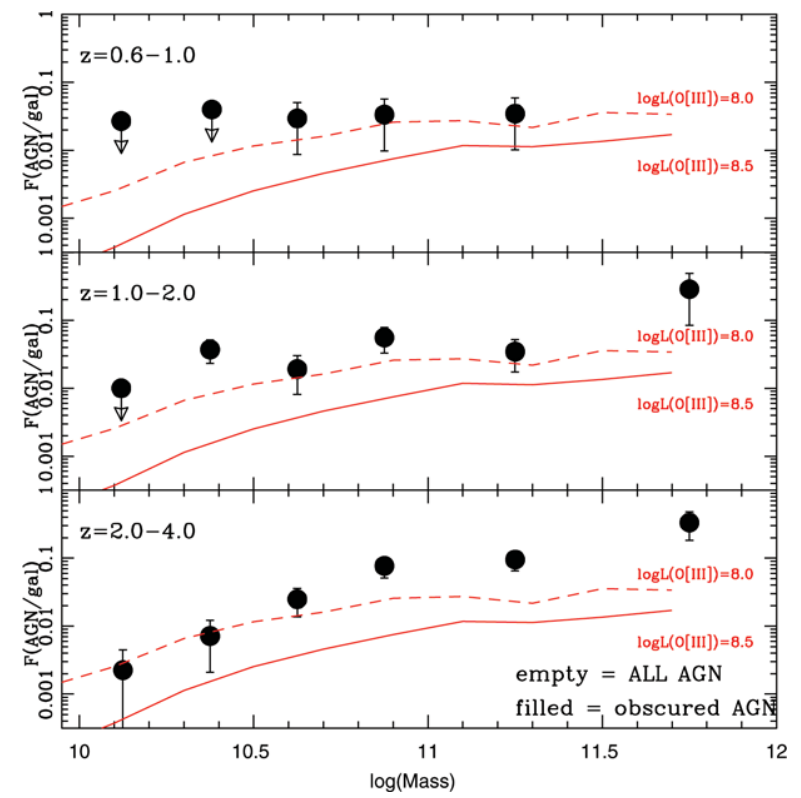

Figure 4. Fraction of obscured AGN with $L_{\mathrm{X}}>10^{43} \mathrm{erg} \mathrm{s}^{-1}$ as a function of the stellar mass in three different redshift bins. Filled circles refer to the "obscured" AGN sample. The dashed (continuous) lines represent the fraction of AGN with $L\left(\mathrm{O}_{\mathrm{III}}\right)>10^{8.0}\left(10^{8.5}\right) L_{\odot}$ in an optically selected sample in the local universe $(z<0.2)$ from SDSS (Best et al. 2005). 
the intrinsic physical properties of AGN hosts, using the unprecedented multiwavelength coverage of the COSMOS field. Merloni et al. (2009) have been able to measure restframe $K$-band luminosities and total stellar masses $M_{*}$ of the hosts of 89 broad-line (unobscured) AGN in the redshift range $1<z<2.2$, for which we measured the black hole mass, $M_{\mathrm{BH}}$, using the virial method (see, e.g., Peterson et al. 2004 and these proceedings). This sample constitutes the largest high-redshift sample so far for which reliable black hole and galaxy masses are available. We find that, compared to the local value, the average black hole to host galaxy mass ratio appears to evolve positively with redshift, with a best fit evolution of the form $(1+z)^{0.68 \pm 0.12}$ (Figure 5$)$. A thorough analysis of observational biases induced by intrinsic scatter in the scaling relations reinforces the conclusion that an evolution of the $M_{\mathrm{BH}}-M_{*}$ relation must ensue for actively growing black holes at early times: either its overall normalization, or its intrinsic scatter (or both) appear to increase with redshift.

\section{Acknowledgements}

It is a pleasure to acknowledge the contribution of Takamitsu Miyaji, Andrea Comastri, Fabrizio Fiore, and Andrea Merloni in obtaining the results I presented at the conference, along with the efforts of many people from the XMM-COSMOS, zCOSMOS and CDFS/MUSIC collaborations. Many thanks to the organizers of the meeting, and in particular Bradley Peterson, for offering me the opportunity to attend this great symposium.

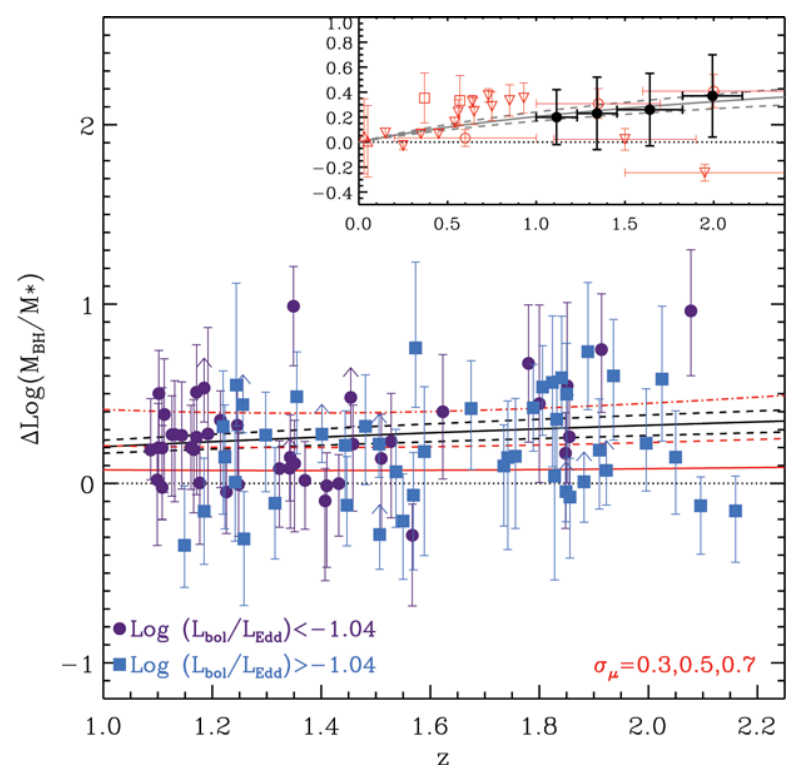

Figure 5. Redshift evolution of the offset measured for zCOSMOS type 1 AGN from the local $M_{\mathrm{BH}}-M_{*}$ relation. Different symbols identify different ranges of Eddington ratios with upwards arrows representing the upper limits on host galaxy mass. The solid black line shows the best fit obtained assuming an evolution of the form $\Delta \log \left(M_{\mathrm{BH}} / M_{*}\right)(z)=\delta(1+z)$ with $\delta=0.68 \pm 0.12$. The solid, dashed, and dot-dashed lines show the bias due to an intrinsic scatter in the scaling relation of $0.3,0.5$ and 0.7 dex, respectively. In the inset, we show a comparison of our data (black circles, binned) with data collected from the literature. 


\section{References}

Aird, J., et al. 2009, MNRAS, in press [arXiv:0910.1141]

Alexander, D. M., et al. 2003, AJ, 126, 539

Alexander, D. M., et al. 2005, ApJ, 632, 736

Antonucci, R. R. \& Miller, J. S. 1985, ApJ, 297, 621

Avni, Y. \& Bahcall, J. N. 1980, ApJ 235, 694

Best, P. N., Kauffmann, G., Heckman, T. M., Brinchmann, J., Charlot, S., Ivezic, Z., \& White, S. D. M 2005, MNRAS, 362, 35

Brandt, W. N. \& Hasinger, G. 2005, ARAA, 43, 827

Brusa, M., et al. 2005, A\&A A, 432, 69

Brusa, M., et al. 2009a, A\&\&A, 507, 1277

Brusa, M., et al. 2009b, ApJ, 693, 8

Comastri, A., et al. 2002, ApJ, 571, 771

Cowie, L. L., Songaila, A., Hu, E. M., \& Cohen, J. G. 1996, AJ, 112, 839

Daddi, E., et al. 2007, ApJ, 670, 156

Donley, J. L., Rieke, G. H., Perez-Gonzalez, P. G., \& Barro, G. 2008, ApJ, 687, 111

Ebrero, J., et al. 2009, A\&A, 493, 55

Fiore, F., et al. 2008, ApJ, 692, 74

Gilli, R., Comastri, A., \& Hasinger, G. 2007, A\&A, 463, 79

Grazian, A., et al. 2006, A\&BA, 449, 951

Hasinger, G. 2008, A\&A, 490, 905

Hickox, R. 2009, Chandra Newsletter, cover article [arXiv:0904.3543]

Hopkins, P. F., Hernquist, L., Cox, T. J., Di Matteo, T., Robertson, B., \& Springel, V. 2006, ApJS, 163, 1

Hopkins, P. F., Hickox, R., Quataert, E., \& Hernquist, L. 2009, MNRAS, 398, 333

Jahnke, K., et al. 2009, ApJ, 706, 215

La Franca, F., et al. 2005, ApJ, 635, 864

Lilly, S. J., et al. 2007, ApJS, 172, 70

Magorrian, J., et al. 1998, AJ, 115, 2285

Maiolino, R., et al. 2007, A\&̈A, 468, 979

Marconi, A. \& Hunt, L. K., 2003, ApJ, 589, L21

Marconi, A., Risaliti, G., Gilli, R., Hunt, L. K., Maiolino, R., \& Salvati, M. 2004, MNRAS, 351, 169

Menci, N., Fiore, F., Puccetti, S., \& Cavaliere, A. 2008, ApJ, 686, 219

Merloni, A. 2004, MNRAS, 353, 1035

Merloni, A., et al. 2009, ApJ, in press [arXiv:0910.4970]

Peng, C. Y., et al. 2006, ApJ, 649, 616

Peterson, B. M., et al. 2004, ApJ, 613, 682

Salvato, M., et al. 2009, ApJ, 690, 1250

Silverman, J. D., et al. 2008, ApJ, 679, 118

Silverman, J. D., et al. 2008, ApJ, 675, 1025

Treister, E. \& Urry, C.M. 2006, ApJ 652, 79

Ueda, Y., Akiyama, M., Ohta, K., \& Miyaji, T. 2003, ApJ 598, 886

Yamada, T., et al. 2009, ApJ, 699, 1354

Woo, J. H., Treu, T., Malkan, M. A., \& Blandford, R. D. 2008, ApJ, 681, 925 